\title{
Characterization of Noncovalent Complexes Formed between Minor Groove Binding Molecules and Duplex DNA by Electrospray Ionization-Mass Spectrometry
}

\author{
David C. Gale* and Richard D. Smith \\ Chemical Sciences Department, Pacific Northwest Laboratory, Richland, Washington, U.S.A.
}

\begin{abstract}
The noncovalent complex formed in solution between minor groove binding molecules and an oligonucleotide duplex was investigated by electrospray ionization-mass spectrometry (ESI-MS). The oligonucleotide duplex formed between two sequence-specific 14-base pair oligonucleotides was observed intact by ESI-MS and in relatively high abundance compared to the individual single-stranded components. Only sequence-specific A:B duplexes were observed, with no evidence of random nonspecific aggregation (i.e., A:A or B:B) occurring under the conditions utilized. Due to the different molecular weights of the two 14-base pair oligonucleotides, unambiguous determination of each oligonucleotide and the sequencespecific duplex was confirmed through their detection at unique mass-to-charge ratios. The noncovalent complexes formed between the self-complementary $5^{\prime}$-dCGCAAATTTGCG-3' oligonucleotide and three minor groove binding molecules (distamycin A, pentamidine, and Hoechst 33258) were also observed. Variation of several electrospray ionization interface parameters as well as collision-induced dissociation methods were utilized to characterize the nature and stability of the noncovalent complexes. The noncovalent complexes upon collisional activation dissociated into single-stranded oligonucleotides and single-stranded oligonucleotides associated with a minor groove binding molecule. ESI-MS shows potential for the study of small molecule-oligonucleotide duplex interactions and determination of small molecule binding stoichiometry. (J Am Soc Mass Spectrom 1995, 6, 1154-1164)
\end{abstract}

$\mathrm{E}$ lectrospray ionization-mass spectrometry (ESIMS) has become an important analytical technique for the characterization of biopolymers [1-5]. Precise molecular weight, sequence, the site and nature of chemical modification, and other structural information can be obtained. One group of biopolymers that can be analyzed readily by ESI-MS are the nucleic acids [6-10]. The polyanionic backbone of nucleic acids enables these molecules to be analyzed by ESI-MS in the negative ion mode. It has been demonstrated recently that protein-protein, protein-ligand, enzyme-substrate, and other important biological noncovalent complexes can be observed by ESI-MS [11-18]. In addition, the specific multimeric association of oligonucleotides has been detected [19-21]. Instrumentally, the compromise between preserving noncovalent complexes formed in solution and obtaining sufficient desolvation to allow effective detection generally re-

Address reprint requests and correspondence to Dr. Richard D. Smith, Chemical Methods and Separations Group, Chemical Sciences Department, Pacific Northwest Laboratories, Battelle Boulevard, P8-19, P.O. Box 999 , Richland, WA 99352.

* Current address: Finnigan MAT, 355 River Oaks Parkway, San Jose, CA 95134. quires careful selection of electrospray ionization (ESI) interface conditions [22].

The interaction and noncovalent binding of small organic molecules to double helix or duplex form DNA provides the basis of many antitumor, antiviral, and antibiotic applications [23-31]. These small organic molecule-oligonucleotide duplex noncovalent complexes have been studied by a number of techniques, which include NMR [32-36], x-ray crystallography [37-40], gel footprinting [41-43], Fourier transform infrared [44], and linear dichroism [45]. A major focus of small molecule-oligonucleotide duplex studies has been the elucidation of structure-function relationships. The eventual goal is to rationally design and synthesize sequence-specific DNA binding small molecules [46-49]. Several different classes of small organic molecules are known to interact with duplex form DNA. One such group is the minor groove binders $[50,51]$. Minor groove binding is characterized by interaction of the bound molecule with the edges of the base pairs in the minor groove of double helix DNA. Hydrogen bonding, van der Waals forces, and electrostatic interactions have been suggested to account for their sequence selectivity $[34,35,52-54]$. The oligonucleotide duplex itself is stabilized by hydrogen 


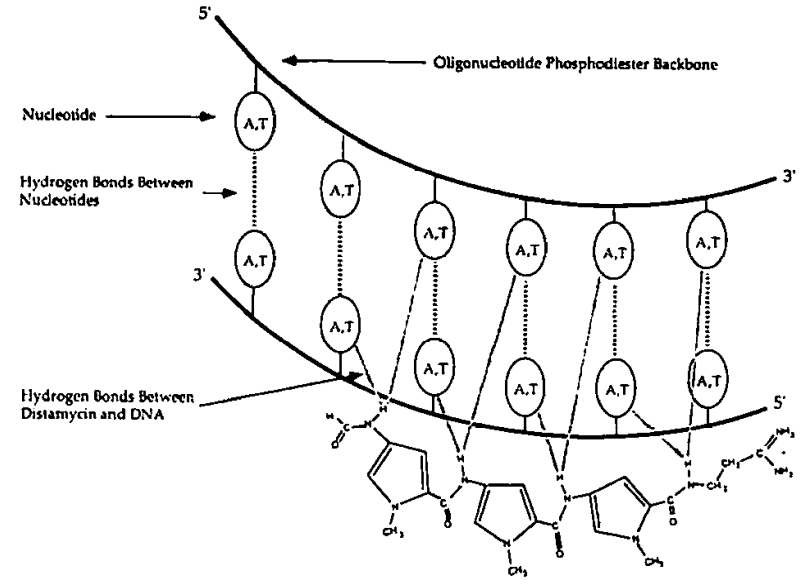

Figure 1. Model for the 1:1 Dm-oligonucleotide duplex noncovalent complex (figure adapted from ref 77 ).

bond formation, hydrophobic interactions (base stacking), and electrostatic forces in the presence of stabilizing counterions $[55,56]$. Distamycin A (Dm), a naturally occurring antibiotic, is a crescent-shaped linear molecule that preferentially binds in the DNA duplex minor groove of four or five successive AT base pairs $[52,57]$. Shown in Figure 1 is a representation of Dm binding to DNA. NMR studies have shown that with the self-complementary $5^{\prime}$-dCGCAAATTTGCG-3' oligonucleotide, both 1:1 and 2:1 Dm-oligonucleotide duplex noncovalent complexes can form in solution, dependent on the concentration of Dm and duplex in solution [34]. The NMR results indicate that the 1:1 Dm-oligonucleotide duplex noncovalent complex forms first. Only after the addition of greater than 1 equiv of $\mathrm{Dm}$, does the 2:1 Dm-oligonucleotide duplex form, where Dm is bound as a side-by-side antiparallel dimer in the minor groove of the duplex (in the AATT or ATTT region) [34]. Association constants for the binding of $\mathrm{Dm}$ to a 16-base pair oligonucleotide duplex and binding of $\mathrm{Dm}$ to a 1:1 Dm-16-base pair oligonucleotide duplex have been measured previously and are $1.3 \times 10^{7}$ and $7.9 \times 10^{8} \mathrm{M}^{-1}$, respectively [58].

Recently we reported [59] the observation by using ESI-MS of the intact noncovalent complex formed between a self-complementary 12-base pair oligonucleotide duplex and Dm. Both 1:1 Dm-oligonucleotide duplex and 2:1 Dm-oligonucleotide duplex noncovalent complexes were observed, dependent on the concentration of Dm. Hsieh et al. [60] utilized ESI-MS to study the binding of actinomycin D-a known DNA duplex binding intercalator-to several single-stranded oligonucleotides. Actinomycin D was observed to bind preferentially to a single-stranded oligonucleotide containing a $5^{\prime}$-dCG-3' sequence. Other than our initial communication, there have been no other reports that utilized ESI-MS to study the interaction between known DNA binding small molecules to duplex form DNA. Numerous studies and reports in the literature have demonstrated that the interaction between $\mathrm{Dm}$ and DNA occurs through the binding of this molecule into the minor groove of double-stranded DNA [34-40, 49-52]. Because of the scientific interest in small molecules that interact with DNA, we have performed additional studies by ESI-MS to determine if this technique can be utilized in the study of these systems. We have extended our studies to include two additional known minor groove binding molecules. We also have investigated the effect of interface heating and performed gas-phase collisional activation on these noncovalent complexes. This article is the first detailed characterization of the noncovalent complex formed between a minor groove binding molecule and duplex form DNA by ESI-MS.

\section{Experimental}

Negative ion ESI mass spectra were acquired with two different quadrupole mass spectrometers. The first instrument has been described previously and only specific instrumental parameters will be noted here [61]. The single quadrupole mass spectrometer used a heated metal capillary interface [62] without countercurrent gas flow. The heated on-axis metal capillary (Alltech, Deerfield, IL) was $20 \mathrm{~cm}$ (15 cm heated) in length with a capillary diameter of $0.50 \mathrm{~mm}$. Individual parameters that were varied for different experiments include the capillary-skimmer potential bias $(\Delta C S)$ and the capillary temperature. The metal capillary was heated resistively by using a power supply (TCR 20S50-1-10T, Electronic Measurements, Neptune, NJ) and the surface temperature of the capillary was measured by using a thermocouple (CN9000A, Omega, Stamford, CT). The application of $\sim 8 \mathrm{~A}(10 \mathrm{~W})$ to the capillary resulted in a surface temperature of $\sim 75^{\circ} \mathrm{C}$. Experimental conditions for data acquisition with this instrument include a sample flow rate of $0.20 \mu \mathrm{L}$ $\min ^{-1}$ [63], an electrospray potential of $-2.4 \mathrm{kV}$, and a coaxial sheath gas of $\mathrm{SF}_{6}$ [64]. The mass spectrometer utilized a Teknivent Vector/Two (Maryland Heights, MO) data system for instrument control and data acquisition.

Negative ion ESI mass spectra were also acquired with a Finnigan MAT TSQ-7000 (San Jose, CA) triple quadrupole mass spectrometer. The electrospray ionization emitter (source) utilized for these studies is identical to the one described previously, which was used in place of the Finnigan MAT emitter. Experimental conditions for all mass spectrometry data from the Finnigan MAT TSQ-7000 include a sample flow rate of $0.20 \mu \mathrm{L} \mathrm{min}^{-1}$, an electrospray potential of $-2.2 \mathrm{kV}$, acquisition time of $2 \mathrm{~min}$, capillary temperature of $120^{\circ} \mathrm{C}$, and a coaxial sheath gas of $\mathrm{SF}_{6}$. Collision-induced dissociation (CID) spectra were acquired by using argon as the collision gas at a pressure of $\sim 1.45 \mathrm{mT}$ and a 5-min acquisition time.

All oligonucleotides utilized in this study were prepared using phosphoramidite chemistry and purified by reversed-phase high-performance liquid chro- 
matography followed by Centricon 3 (Amicon, Beverly, MA) filtration. The purified oligonucleotides were diluted in water (typically by 10) and analyzed by ESI-MS for failure sequence content and degree of sodium adduction. If the failure sequence content was $>5 \%$ or showed $>3$ or 4 sodium adducts for any charge state, the oligonucleotide was repurified. The concentration of oligonucleotide varied from 20 to 75 $\mu \mathrm{M}$ (measured as single-stranded material). Distamycin $\left(\mathrm{Dm}, M_{\mathrm{r}}=481.5\right)$, pentamidine $\left(\mathrm{Pm}, \mathrm{M}_{\mathrm{r}}=\right.$ 340.2), and Hoechst $33258\left(\mathrm{Ht}, M_{r}=424.4\right)$ were purchased from Sigma Chemical (St. Louis, MO) and used without further purification. Oligonucleotide samples were annealed by heating to $95{ }^{\circ} \mathrm{C}$ for $5 \mathrm{~min}$ and cooling to room temperature over $3 \mathrm{~h}$, unless otherwise noted. Solution conditions for oligonucleotide duplex formation involved buffers of either ammonium acetate or a mixture of ammonium citrate-ammonium acetate.

\section{Results and Discussion}

The study of noncovalent complexes by ESI-MS first requires determining effective emitter, ESI interface, and instrumental conditions [22]. Ideally, only those noncovalent complexes that exist in solution will be observed in the mass spectrum. Experimental conditions that lead to nonspecific aggregation (i.e., high concentrations, slow droplet desolvation, etc.) were avoided in these studies. It has been reported that nonspecific dimers have been observed at elevated analyte concentrations from solutions that contain no stabilizing counterions [65-67]. For the majority of the work reported here, the oligonucleotide concentration was $<100 \mu \mathrm{M}$. Shown in Figure 2 is the ESI mass spectrum of the self-complementary $5^{\prime}$-dCGCAAATTTGCG-3' oligonucleotide in unbuffered aqueous solution (concentration $75 \mu \mathrm{M} ; M_{r}=3645.5$ ). The mass spectrum was acquired by using gentle interface conditions $(\Delta \mathrm{CS}=-100 \mathrm{~V}$, capillary heating of $\sim 10 \mathrm{~W}$, capillary temperature of $75^{\circ} \mathrm{C}$ ). A charge state distribution that corresponds to only the single-stranded oligonucleotide is observed as expected. The instru-

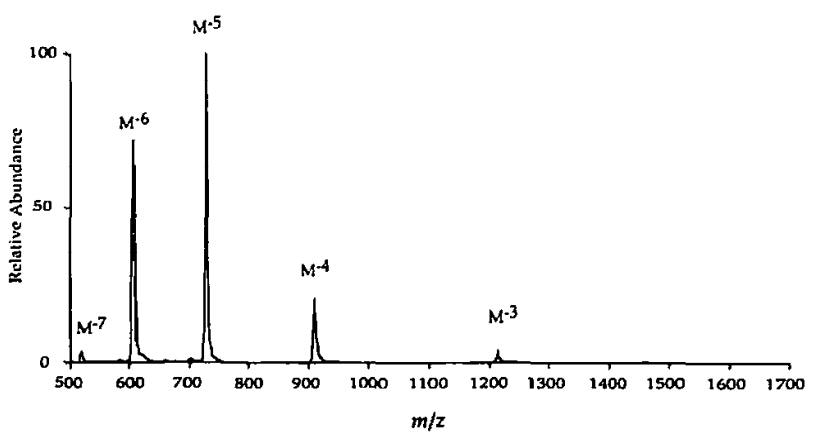

Figure 2. Negative ion electrospray mass spectrum of $5^{\prime}$ dCGCAAATTTGCG-3' in aqueous solution only, acquired with the single quadrupole instrument. mental conditions used to acquire Figure 2 were the same as those used previously to observe noncovalent complexes of this self-complementary oligonucleotide (i.e., low capillary-skimmer potential bias and capillary temperatures) [59]. In a solution without the stabilizing counterions, oligonucleotide duplex noncovalent complexes are not stable in solution and are not observed. No dimers, trimers, tetramers, or other contributions due to random aggregation or nonspecific association during the ESI process are observed. This experiment provides a necessary and convincing control for studies in which noncovalent complexes exist in solution at similar ESI-MS conditions and oligonucleotide concentrations.

Self-complementary oligonucleotides are attractive for these studies due to spectral simplicity and the need for reduced sample preparation and purification. However, assignment of a peak due to either singlestranded or duplex species in the ESI mass spectrum of an annealed self-complementary oligonucleotide in buffer can be complicated. Both single-stranded and duplex oligonucleotide ions with even charge states can have the same mass-to-charge ratio, where the duplex has twice the charge of the single-stranded oligonucleotide (monomer). For example, the selfcomplementary 5' -dCGCAAATTTGCG-3' oligonucleotide, both (single-stranded oligonucleotide) ${ }^{-3}$ and (duplex $)^{-6}$ ions have the same nominal mass-to-charge ratio value (1214). Odd charge states of self-complementary oligonucleotide duplexes are assigned easily due to their unique mass-to-charge ratio. In Figure 3, the peaks at $m / z 1040\left(\Delta^{-7}\right)$ and $1457\left(\Delta^{-5}\right)$ are odd charge states specific to the oligonucleotide duplex $\left(M_{\mathrm{r}}=7290\right)$. However, peaks at $m / z 1214$ and 1822 may include contributions from both single-stranded and duplex oligonucleotide ions due to the same massto-charge ratio of even charge states. One possible method for assignment of these ions is based on the incremental mass-to-charge ratio difference of known adducts. Inspection of the spectrum showed a spacing of $m / z 5.6 \pm 0.3$ units between the peak at $m / z 1822$

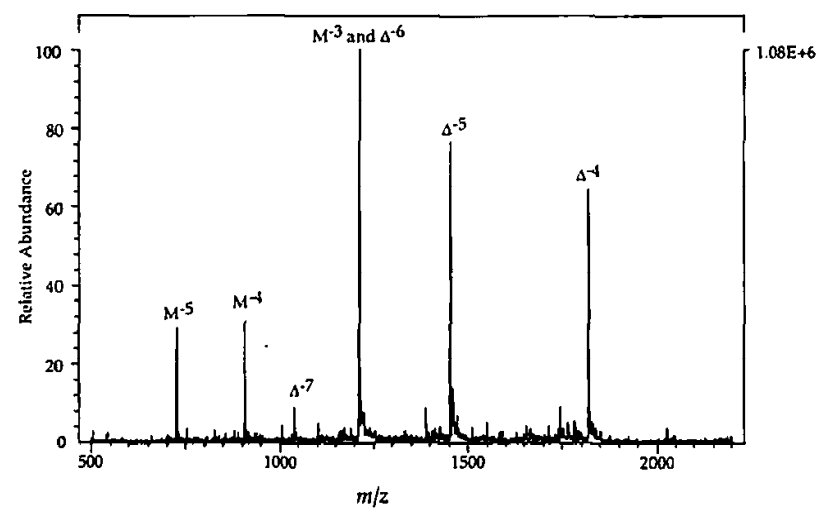

Figure 3. Negative ion electrospray mass spectrum of $5^{\prime}$ dCGCAAATTTCCG-3' $(75 \mu \mathrm{M})$ annealed in 20-mM ammonium acetate $(\mathrm{pH} \sim 8.1)$ acquired with the Finnigan MAT TSQ-7000 instrument at a capillary temperature of $120^{\circ} \mathrm{C}$. 
and the first adduct peak. Assignment of the peak at $m / z 1822$ to the $4-$ charge state of the duplex $\left(\Delta^{-4}\right)$ enables an adduct mass of approximately $22 \mathrm{u}$ to be calculated. An adduct of $22 \mathrm{u}$ corresponds to the molecular weight difference for substitution of sodium for a hydrogen, a typical contaminant in oligonucleotide samples. Therefore, the peak at 1822 can be identified with a high degree of certainty as the $4-$ charge state of the duplex. The peak at $m / z 1214$ is believed to be comprised of both single-stranded and duplex ions, based on the observation of the duplex at $m / z 1040$ and observation of an intense signal at $\mathrm{m} / \mathrm{z}$ 910 for the 4 - charge state of the single-stranded oligonucleotide. Observation of single-stranded oligonucleotide peaks at lower mass-to-charge ratio (i.e., $m / z<1000$ ) may be due to incomplete duplex formation or the dissociation of high charge state duplex ions in the electrospray interface [20]. The latter is less likely because the average charge state for duplex ions is expected to be lower and to yield lower charge state monomers (compare Figures 2 and 3 ).

Generally, the effective formation of oligonucleotide noncovalent complexes in solution requires annealing. The heating and gradual cooling process results in formation of the most stable small moleculeoligonucleotide duplex conformation. In this study we investigated the effect of the annealing process on the Dm-oligonucleotide noncovalent complex. Shown in Figure 4 are mass spectra obtained under different interface and solution conditions: before annealing the solution by using gentle interface conditions (Figure 4a), after annealing by using gentle interface conditions (Figure $4 \mathrm{~b}$ ), and after annealing by using very energetic interface conditions, as used in more conventional ESI-MS studies (Figure 4c). The concentration of $\mathrm{Dm}$ in this experiment (Figure $4 \mathrm{a}-\mathrm{c}$ ) was adjusted to twice that of the oligonucleotide duplex.

The nonannealed solution (Figure 4a) contains a number of different charge state distributions, as evidenced by unique molecular weight or odd charge state ions. The presence of stabilizing counterions enables the formation of several noncovalent species, which include both 1:1 and 2:1 Dm-oligonucleotide duplex noncovalent complexes, without annealing. There are, however, a large number of peaks that correspond to single-stranded oligonucleotides; these may arise from the formation of less stable complexes (i.e., base pair mismatched) that are dissociated more readily in the ESI interface. There is also a significant component that corresponds to the single-stranded oligonucleotide with a Dm adduct. Unique identifiable odd charge state peaks include 1:1 Dm-oligonucleotide duplex (i.e., $[\Delta: 1 \mathrm{Dm}]^{-7}$ ), 2:1 Dm-oligonucleotide duplex (i.e., $[\Delta: 2 \mathrm{Dm}]^{-7}$ ), low charge state singlestranded oligonucleotides (i.e., $\mathrm{M}^{-3}$ ), and singlestranded oligonucleotides with a Dm adduct (i.e., [M $+D m]^{-5}$ ). These results indicate that small molecule-oligonucleotide duplex noncovalent complexes can form prior to annealing. Peaks that may

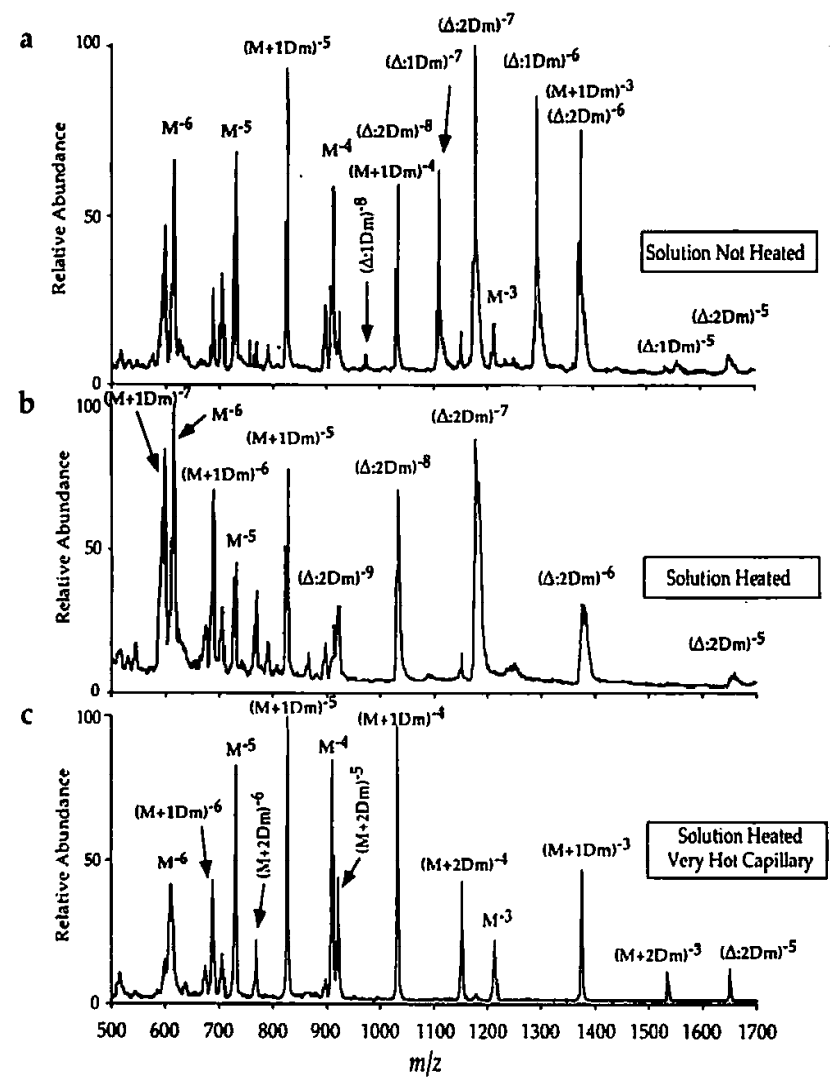

Figure 4. Negative ion ESI mass spectra of $20-\mu \mathrm{M} \mathrm{Dm}-20-\mu \mathrm{M}$ $5^{\prime}$-dCGCAAATTTGCG-3' in 10-mM ammonium acetate-10-mM ammonium citrate $(\mathrm{pH} \sim 8.3)$. Peaks labeled $M$ are singlestranded oligonucleotide, $\Delta$ are oligonucleotide duplex, $(\Delta: 1 \mathrm{Dm})$ are 1:1 Dm-duplex, $(\Delta: 2 \mathrm{Dm})$ are $2 ; 1 \mathrm{Dm}$-duplex, $(\mathrm{M}+\mathrm{Dm})$ are single-stranded oligonucleotide with one $\mathrm{Dm}$, and $(M+2 \mathrm{Dm})$ are single-stranded oligonucleotide with two $\mathrm{Dm}$. Individual conditions for each mass spectra are as follows: (a) not annealed, $\triangle C S$ of $-100 \mathrm{~V}$, capillary heating of $-10 \mathrm{~W}\left(\sim 75^{\circ} \mathrm{C}\right)$; (b) annealed, instrumental conditions the same as in (A); (c) annealed, $\triangle C S$ of $-125 \mathrm{~V}$, capillary heating of $-12 \mathrm{~W}\left(-105^{\circ} \mathrm{C}\right)$.

have contributions from two different ions are noted in Figure $4 \mathrm{~A}$ by placing both labels above the peak. For example, the peak at $m / z 1031$ may have contributions from both $[\Delta: 2 \mathrm{Dm}]^{-8}$ and $[\mathrm{M}+\mathrm{Dm}]^{-4}$. The observation of single-stranded oligonucleotides with $\mathrm{Dm}$ in Figure $4 \mathrm{a}$ is not surprising, given the possibility of hydrogen bonding between Dm and single-stranded oligonucleotides. In addition, there may be an electrostatic attraction between the positively charged Dm and the negatively charged oligonucleotide. However, numerous studies in the literature have demonstrated that $\mathrm{Dm}$ binding in the minor groove of doublestranded DNA is the biologically significant interaction for this molecule.

Electrospraying the annealed solution results in the observation, at higher mass-to-charge ratio, of a charge state distribution for the 2:1 Dm-oligonucleotide duplex noncovalent complex as evidenced by unique odd charge state peaks (i.e., $[\Delta: 2 \mathrm{Dm}]^{-7}$ ). These data are shown in Figure $4 b$. This result is consistent with NMR 
data for the $\mathrm{Dm}$ to oligonucleotide duplex concentration. After annealing the solution, no peaks due to single-stranded oligonucleotides with a $\mathrm{Dm}$ are observed at high mass-to-charge ratio (Figure $4 \mathrm{~b}$ ). These results suggest that the 2:1 Dm-oligonucleotide duplex noncovalent complex is the most stable conformation given the $\mathrm{Dm}$ concentration and oligonucleotide sequence. In addition, these results demonstrate that Dm has a significant binding affinity to this oligonucleotide duplex. The differences observed between Figure $4 \mathrm{a}$ and $\mathrm{b}$ are due solely to the annealing process. Ions due to both single-stranded oligonucleotides and single-stranded oligonucleotides associated with $\mathrm{Dm}$, observed at lower mass-to-charge ratio in Figure 4b, may arise from incomplete or mismatched duplex formation or the dissociation of higher charge state ions of the 2:1 Dm-oligonucleotide duplex noncovalent complex in the ESI interface [20]. No 1:1 Dmoligonucleotide duplex (i.e., $[\Delta: \mathrm{Dm}]^{-7}$ ) or low charge state single-stranded oligonucleotide ions (i.e., $\mathrm{M}^{-3}$ ) are observed in Figure $4 b$, unlike the nonannealed solution (Figure 4a).

Greater activation in the ESI interface causes the 2:1 $\mathrm{Dm}$-oligonucleotide duplex ions to be largely dissociated (with the exception of the 5- charge state at higher mass-to-charge ratio). The 2:1 Dm-oligonucleotide duplex is dissociated into single-stranded oligonucleotides and single-stranded oligonucleotides with varying amounts of Dm (Figure 4c), which give rise to additional peaks in the higher mass-to-charge ratio range. Peaks that apparently arise from the breakup of the 2:1 Dm-oligonucleotide duplex in the ESI interface include single-stranded oligonucleotides with a Dm adduct (i.e., $[\mathrm{M}+\mathrm{Dm}]^{-4}$ ), single-stranded oligonucleotides with two $\mathrm{Dm}$ adducts (i.e., $[\mathrm{M}+2 \mathrm{Dm}]^{-4}$ ), and single-stranded oligonucleotides (i.e., $\mathrm{M}^{-3}$ ).

Oligonucleotides of different molecular weight that are sequence specific (i.e., complementary) circumvent the ambiguities associated with the use of selfcomplementary oligonucleotides, because the two single-stranded oligonucleotides and the sequence-specific duplex have different mass-to-charge ratio values. Shown in Figure 5 are the ESI mass spectra obtained for an annealed solution that contained two sequencespecific 14-base pair oligonucleotides. The molecular weight of the duplex is $8528 \mathrm{u}$. Charge state distributions for the two single-stranded oligonucleotides, strand $C$ and strand $G$, are observed along with a charge state distribution for the oligonucleotide duplex that consists of one $C$ strand and one $G$ strand $(C: G=$ $\Delta)$. With very gentle interface conditions, broad peaks due to a distribution of putatively solvated and/or adducted ions are detected (Figure $4 \mathrm{a}$ ). The adducting species may include water, ammonium ions, sodium ions, and other counterions. The gentleness of the interface conditions enables the most effective detection of the intact noncovalent complex, but generally results in reduced sensitivity. With increased capillary heating and a larger capillary skimmer bias $(\Delta C S)$, ions

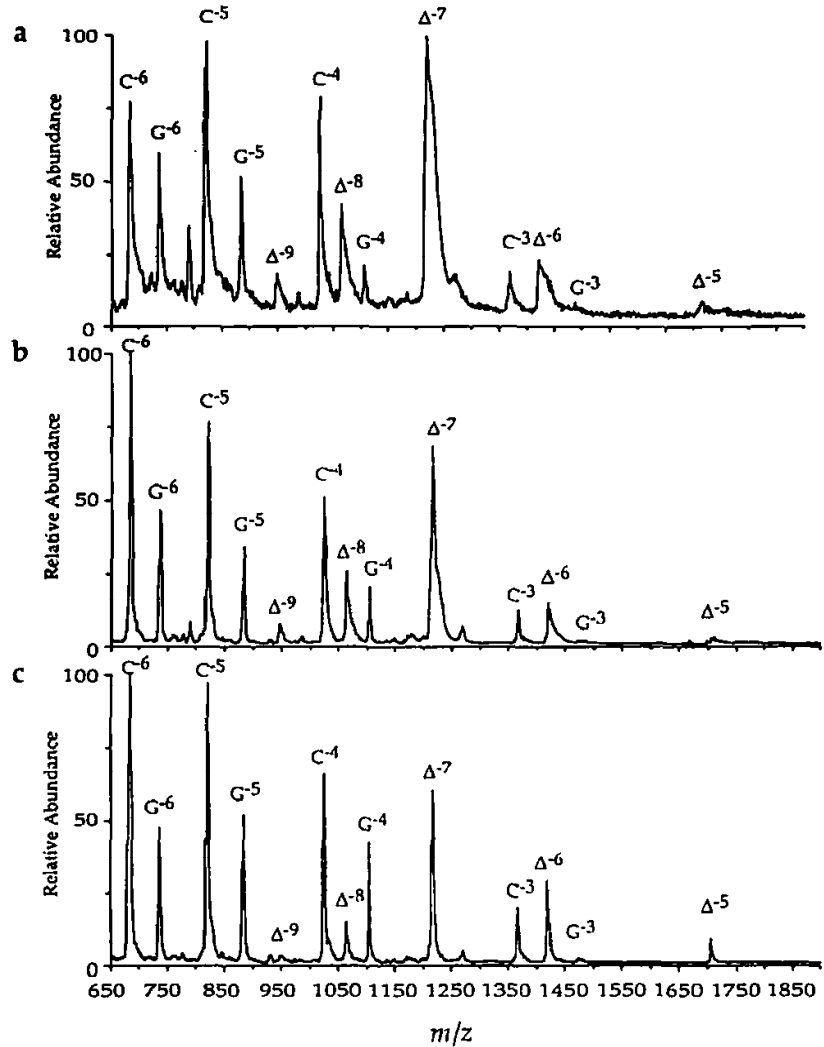

Figure 5. Comparison of negative ion ESI-mass spectra of $20-\mu \mathrm{M}$ $5^{\circ}$-dCCCCAAATTTCCCC-3' (strand C, $M_{r}=4104$ ) and $20-\mu \mathrm{M}$ $5^{\prime}$-dGGGGAAA TTTGGGG-3' (strand $G, M_{r}=4424$ ) solution, annealed in 10-mM ammonium citrate-10-mM ammonium acetate, $\mathrm{pH} \mathrm{8.3,} \mathrm{acquired} \mathrm{under} \mathrm{(a)} \mathrm{extremely} \mathrm{gentle} \mathrm{interface} \mathrm{conditions}$ ( $\triangle \mathrm{CS}$ of $-50 \mathrm{~V}$, capillary heating of $\sim 10 \mathrm{~W}, \sim 75{ }^{\circ} \mathrm{C}$ ), (b) gentle interface conditions ( $\triangle \mathrm{CS}$ of $-100 \mathrm{~V}$, capillary heating of $\sim 10$ $W$ ), and (c) harsh interface conditions ( $\triangle \mathrm{CS}$ of $-100 \mathrm{~V}$, capillary heating of $\sim 12 \mathrm{~W}, \sim 105^{\circ} \mathrm{C}$ ). Peaks labeled $\mathrm{C}, \mathrm{G}$, and $\mathrm{J}$ arise from the two single-stranded species and the sequence specific oligonucleotide duplex (C:G), respectively.

are detected with only minor adduction (Figure $5 b$ and c). A decrease in the relative abundance of the noncovalent complex species in the gas phase is evident by a comparison of the relative abundances for the $\Delta^{-7}$ oligonucleotide duplex noncovalent complex ion and $\mathrm{C}^{-4}$ single-strand oligonucleotide ions (Figure $5 \mathrm{a}-\mathrm{c}$ ). With an increase in interface energy, the intensity of $\Delta^{-7}$ (the oligonucleotide duplex) relative to the intensity of $\mathrm{C}^{-4}$ is decreased. Also, the increase in interface excitation has a detrimental effect on the observation of high charge state duplex ions (e.g., the $\Delta^{-9}$ peak). At gentle interface energy conditions, the $\Delta^{-9}$ species is present in mach greater abundance, but under harsher interface conditions it has disappeared; presumably it has dissociated into single-stranded ions. The harsher interface conditions employed in Figure $5 c$, although they are energetic enough to decrease the abundance of the noncovalent complex, are insufficient to induce detectable dissociation of the oligonucleotide covalent bonds. 
For all interface conditions, charge state distributions for the duplex are shifted to lower charge state (higher mass-to-charge ratio) than the charge state distributions for the single-stranded oligonucleotides. The reduced charging for oligonucleotide complexes has been observed previously and may be due to several factors, which include breakup of higher charge states of the duplex in the interface region or the displacement of negative charges from the anionic backbone by ion molecule reactions during transfer to the gas phase (driven by structural and/or coulombic constraints for a more compact structure) [12]. The only peaks in Figure 5 that are not due to either the single-stranded $C$, single-stranded $G$, or duplex (C:G) species, are those at $m / z 786$ and 1270 . The peak at $m / z 786$ (Figure 5a) corresponds to a citrate cluster species. This particular peak is not observed in Figure $5 c$, where the increased energy induces its dissociation to lower mass-to-charge ratio species. The small contribution evident at $m / z 1270$ might be due to two $G$ strands in a hairpin-type structure with two $\mathrm{Na}$ or one $\mathrm{K}$ ion to form a quadruplex-type noncovalent complex. No corresponding contribution for two $C$ strands is observed. Oligonucleotides with several consecutive $G$ base pairs are known to form these types of DNA structures [68, 69] and have been previously observed by mass spectrometry [70].

The tandem quadrupole mass spectrometer allows the oligonucleotide duplex ions to be dissociated in the gas phase, which potentially provides information on the distribution of charges between the two 14-base pair oligonucleotides that comprise the noncovalent complex. The product ion spectra for dissociation of the $7-$ and 6 - charge states of the duplex are shown in Figure 6. A wide mass range was scanned to ensure that all charge states of the dissociated duplex would be observed. Dissociation of the oligonucleotide duplex ions results in the observation of ions that correspond to the single-stranded oligonucleotides that comprise the duplex. The spectrum for the $\Delta^{-7}$ species yields primarily the $\mathrm{C}^{-4}, \mathrm{C}^{-3}, \mathrm{G}^{-4}$, and $\mathrm{G}^{-3}$ products. The product ion spectrum of the $\Delta^{-6}$ species results in the observation of $\mathrm{C}^{-3}$ and $\mathrm{G}^{-3}$ products almost exclusively. These results indicate that the charge observed on the electrosprayed duplex ions is distributed equally on both of the oligonucleotides in the gas phase [20].

The duplex formed by the two 14-base pair oligonucleotides has a minor groove binding site, for which Dm has a high binding affinity - specifically the center region of the two oligonucleotides that contains the $5^{\prime}$-dAAATTT-3' sequence [52]. Shown in Figure 7 are the two sequence-specific 14-base pair oligonucleotides after addition of Dm to the solution (at a Dm to duplex ratio of approximately 0.3 ). Charge state distributions for the two single-stranded oligonucleotides, along with a charge state distribution for the oligonucleotide duplex and the 1:1 Dm-oligonucleotide duplex noncovalent complex, are evident in Figure 7. It is important
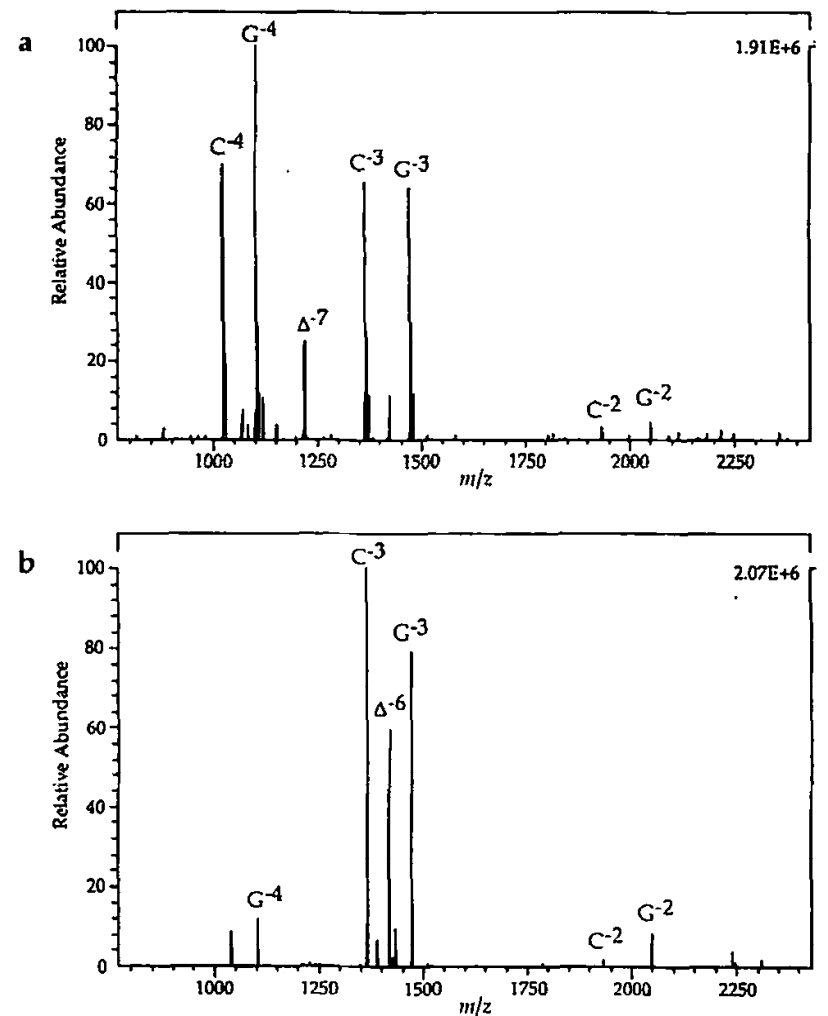

Figure 6. Product ion mass spectra for (a) 7 - (140-eV collision energy) and (b) 6 - (120-eV collision energy) charge states of the duplex formed from the oligonucleotides and solution conditions given in Figure 4. All spectra were acquired with $1.42-\mathrm{mT}$ argon collision gas.

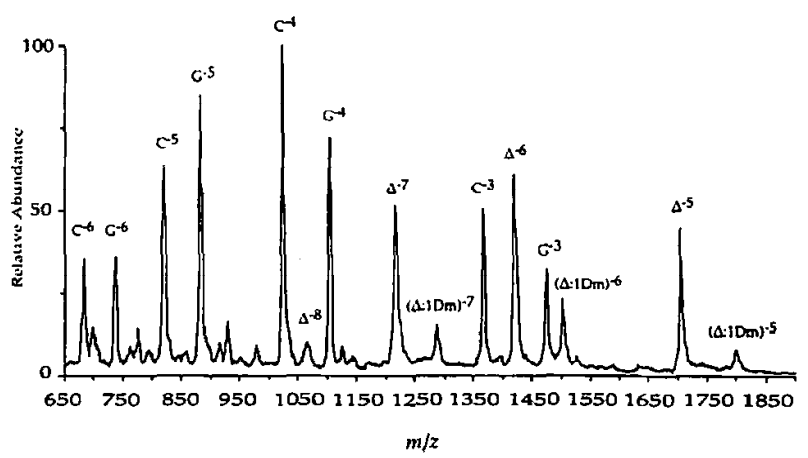

Figure 7. Negative ion electrospray mass spectrum of the same two 14-base pair oligonucleotides and solution conditions used in Figure 4, after the addition of $\operatorname{Dm}(6 \mu \mathrm{M})$. Acquired with the single quadrupole instrument at a $\triangle C S$ of $-125 \mathrm{~V}$ and capillary heating of $\sim 12 \mathrm{~W}\left(\sim 105^{\circ} \mathrm{C}\right)$. Peaks labeled $\Delta$ and $\Delta: 1 \mathrm{Dm}$ correspond to the sequence-specific oligonucleotide duplex $(C: G)$ and the duplex complexed with the minor groove binding molecule Dm, respectively.

to note that only the noncovalent complex composed of one $C$ strand and one $G$ strand is observed. No nonspecific dimers (i.e., $C: C$ or $G: G$ ) are observed complexed with the minor groove binding molecule. A comparison of the oligonucleotide charge state distributions observed in Figures $5 c$ and 7 reveals two noteworthy points. First, the relative abundance of 
higher charge states for single-stranded oligonucleotides (i.e., $\mathrm{C}^{-6}, \mathrm{G}^{-6}$ ) are less intense in Figure 7 than in Figure 5. Second, lower charge states for noncovalent complexes (i.e., $\Delta^{-5}$ ) have greater relative abundance in Figure 7 when compared to Figure 5c. In part, these differences may be due to the greater capillary-skimmer bias used to acquire the spectrum shown in Figure 7 compared to Figure 5c, or may reflect the slightly different solution conditions. However, from these observations it appears that the noncovalent complex that incorporates the minor groove binder is more stable in the gas phase.

A number of molecules have been shown to interact with duplex DNA through minor groove interactions $[37,50]$. Shown in Figure 8 are ESI-MS mass spectra for three different minor groove binding molecules: distamycin (Figure 8a), pentamidine (Figure 8b), and Hoechst 33258 (Figure 8c), with the self-complementary duplex. The peak at $\mathrm{m} / \mathrm{z} 1214$ likely arises from contributions from both single-stranded and duplex oligonucleotides. A distinctive mass-to-charge ratio shift is observed in each case due to the minor groove binding molecules. At the concentration of minor groove binding molecule to oligonucleotide used (approximately 0.6 to 1 ), noncovalent complexes of the duplex with two minor groove binding molecules are not expected. This is supported by the absence of any unique odd charge states (5-) for the 2:1 minor groove

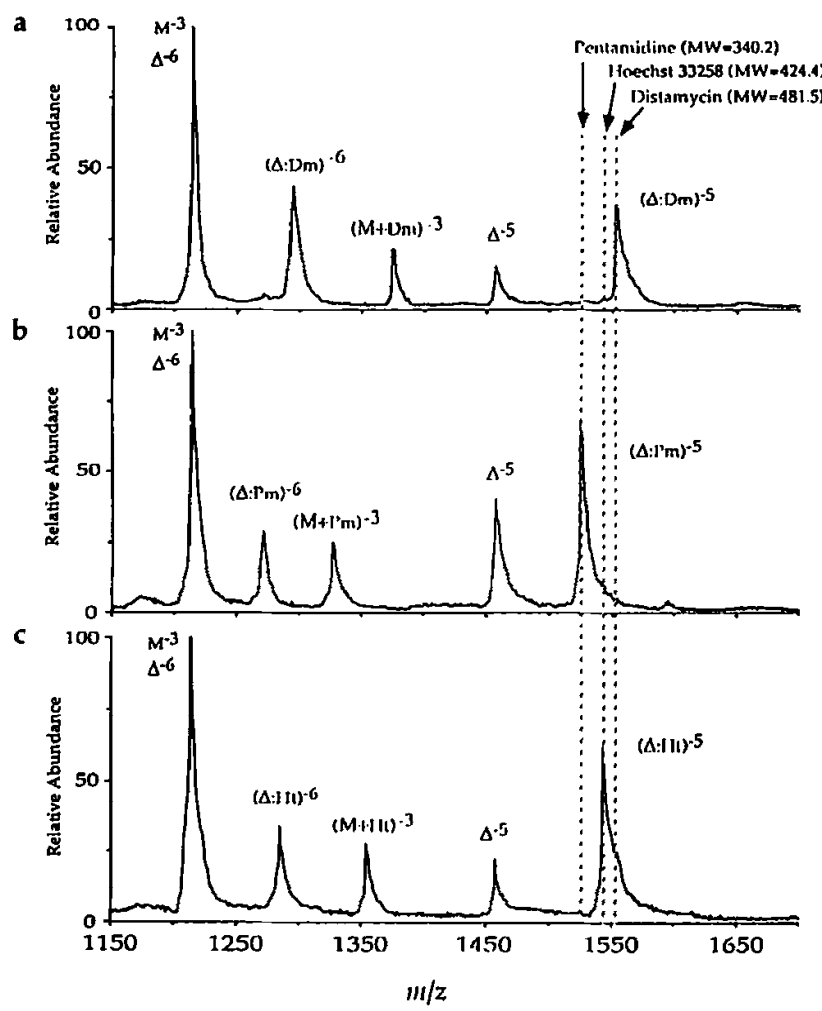

Figure 8. Negative ion electrospray mass spectra of 5'dCGCAAATTTGCG-3' in 30-mM ammonium acetate (pH $~ 8.1$ ) with three different minor groove binding molecules, distamycin (a), pentamidine (b), and Hoechst 33258 (c), acquired with the single quadrupole instrument under similar interface conditions. binding molecule-oligonucleotide duplex. This observation enables the assignment of peaks at $m / z 1374$ (Figure 8a), $m / z 1327$ (Figure $8 b$ ), and $m / z 1355$ (Figure $8 c$ ) as arising from the single oligonucleotide strand with the minor groove binding molecule as an adduct, and not as a 2:1 minor groove binding molecule-oligonucleotide duplex. These ions most likely arise from the breakup of 1:1 minor groove binding molecule-oligonucleotide duplex noncovalent complexes in the ESI interface region. Differences in the relative intensities of the minor groove binding molecule-oligonucleotide duplex ions in Figure 8 may reflect uncertainties in preparation of the small samples used in this work, as well as small differences in the optimal ESI interface conditions, and day-to-day variations in instrumental performance, rather than differences in the dissociation of the complexes in the ESI interface.

The dissociation of the noncovalent complexes was studied to qualitatively investigate their gas-phase stability and to determine whether differences in solution binding affinity of the minor groove binders was reflected in their gas-phase stability. Product ion spectra for collision-induced dissociation of the oligonucleotide duplex noncovalent complexes are shown in Figure 9. Experimental conditions utilized to acquire the spectra were identical including oligonucleotide and ammonium acetate concentration. The nonadducted 5 - charge state of each noncovalent complex was examined, due to their relatively large abundance in the mass spectra and the absence of potential contributions due to monomer species. Shown in Figure $9 a$ is the product ion spectrum of the oligonucleotide duplex, $m / z 1456\left(\Delta^{-5}\right)$. As expected, the oligonucleotide duplex dissociates into single-stranded oligonucleotides $\left(\mathrm{M}^{-3}\right.$ and $\left.\mathrm{M}^{-2}\right)$. Dissociation of the minor groove binding molecule-oligonucleotide duplexes (Figure 9b, $c$, and d) results in the observation of several ions, specifically the 3 - and 2 - charge states of the single-stranded oligonucleotides, and the 3and 2 - charge states of the single-stranded oligonucleotides with a minor groove binding molecule. The only case where this was not observed was for the dissociation of the 1:1 $\mathrm{Ht} /$ oligonucleotide duplex, where the $[\mathrm{M}+\mathrm{Ht}]^{-2}$ ion was not detected (Figure $9 \mathrm{~d}$ ). These results show that the 1:1 minor groove binding molecule-oligonucleotide duplex noncovalent complexes are dissociated easily. These results also indicate that the bare oligonucleotide duplex dissociates more readily than the three minor groove binding-oligonucleotide duplex complexes. The similarity of the dissociation behavior may be attributed to the absence of significant binding differences between the three minor groove binding molecules and/or that the dissociation behavior is governed primarily by the oligonucleotide duplex, which in this experiment remained constant.

Of recent interest concerning minor groove binding has been the ability of these molecules to bind as a 


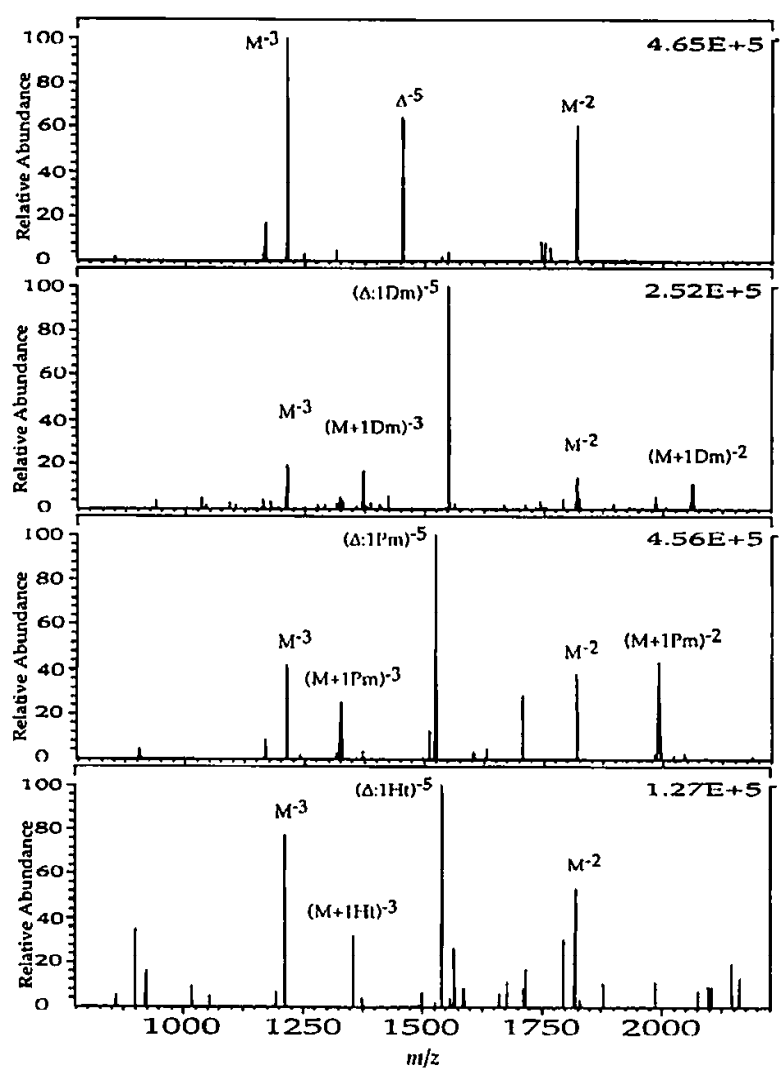

Figure 9. Product ion mass spectra of the self-complementary oligonucleotide, 5' -dCCCAAATTTGCG-3', in 30-mM ammonium acetate $(\mathrm{pH}-8.3)$ with three different minor groove binding molecules. All spectra were acquired with $100-\mathrm{eV}$ collision enargy and 1.40-mT argon collision gas. Product ion mass spectra for $(a)$ the $\Delta^{-5}$ ion, (b) the $(\Delta: 1 \mathrm{Dm})^{-5},(\mathrm{c})$ the $(\Delta: \mathrm{Pm})^{-5}$, and $(\mathrm{d})$ the $(\Delta: H t)^{-5}$ ion.

dimer in the minor groove of duplex DNA [71-77]. The simultaneous binding of two minor groove binding molecules has been exploited by several groups seeking to rationally design enhanced binding affinity to mixed (A-T and $C-G$ ) [72-77] and $C-G$ [71] oligonucleotide sequences. NMR experiments have shown that the binding of two Dm molecules in the minor groove of the $5^{\prime}$-dCGCAAATTTGCG-3' oligonucleotide duplex results in an expansion of the minor groove by $\sim 2.8 \AA$ [34]. In addition, the solution binding affinities of $\mathrm{Dm}$ to an oligonucleotide duplex and Dm to a 1:1 oligonucleotide duplex noncovalent complex are known [58]. With binding affinity and structural differences between the 1:1 and 2:1 Dm-oligonucleotide duplex noncovalent complexes, it is of interest to determine if differences can be observed in the gas-phase dissociation of these complexes.

Shown in Figure 10 are product ion spectra for the 1:1 Dm-oligonucleotide duplex (Figure 10a, b, and c) and for the 2:1 Dm-oligonucleotide duplex (Figure 10d, e, and f) obtained by using different collision energies. The 5 - charge state was utilized as the precursor ion for both the $1: 1$ and 2:1
Dm-oligonucleotide duplex noncovalent complexes: Experimental conditions for tandem mass spectrometry studies of both noncovalent complexes were identical. The only solution difference was the concentration of the minor groove binding molecule Dm. For the dissociation of the 1:1 Dm-oligonucleotide duplex, only one Dm adduct along with the single-stranded oligonucleotide was observed. For the dissociation of the 2:1 Dm-oligonucleotide duplex, ions with both one and two Dm adducts for a single oligonucleotide are observed along with single-stranded oligonucleotide ions. No 1:1 Dm-oligonucleotide duplex was observed from the dissociation of the 2:1 Dm-oligonucleotide duplex. In addition, no bare oligonucleotide duplex is observed from the dissociation of either the $1: 1$ or $2: 1$ Dm-oligonucleotide duplex. At lower collision energies the parent noncovalent complex ion remains the most abundant ion. Both the $1: 1$ and $2: 1$ Dm-oligonucleotide duplex complexes dissociate to yield intact single-stranded oligonucleotide and single-stranded oligonucleotide with $\mathrm{Dm}$ adduct ions. Dissociation of the 2:1 Dm-oligonucleotide duplex also results in the observation of single-stranded oligonucleotide with two Dm.

In this experiment, the single-stranded oligonucleotide with $\mathrm{Dm}$ ions results from dissociation of the $\mathrm{Dm}$-oligonucleotide duplex noncovalent complex. It is known that $\mathrm{Dm}$ is buried in the minor groove of the
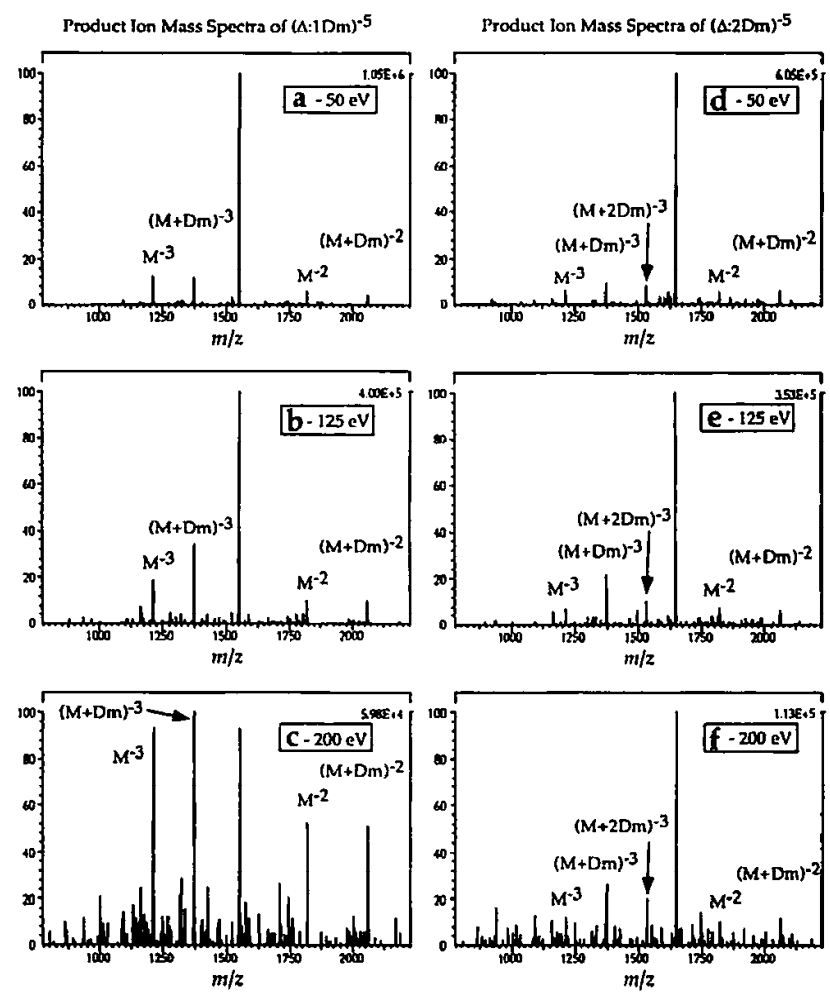

Figure 10. Product ion mass spectra for the 5 - charge states of 1:1 (a, $50 \mathrm{eV} ; \mathrm{b}, 125 \mathrm{eV} ; \mathrm{c}, 200 \mathrm{eV}$ ) and 2:1 (d, $50 \mathrm{eV}$; e, $125 \mathrm{eV} ; \mathrm{f}$, $200 \mathrm{eV}) \mathrm{Dm}$-oligonucleotide duplex complexes in 30 -mM ammonium acetate $(\mathrm{pH} \sim 8.1)$ at different collision energies. All spectra were acquired with $1.40-\mathrm{mT}$ argon collision gas. 
oligonucleotide duplex and that it forms hydrogen bonds to the nucleotides that comprise the oligonucleotide duplex [34, 37, 40]. The surface area of the oligonucleotide duplex should be greater than that of the $\mathrm{Dm}$ buried in the minor groove. Therefore, the oligonucleotide duplex will undergo most if not all of the collisions. Dissociation of the Dm-oligonucleotide duplex may begin at one of the duplex ends, which results in the "unzipping" of the double helix structure. As the double helix is "unzipped," the hydrogen-bonded $\mathrm{Dm}$ may remain noncovalently bound to one of the now two single-stranded oligonucleotides. If the single-stranded oligonucleotide with Dm undergoes additional collisions, dissociation between the oligonucleotide and Dm would occur (CID of single-stranded oligonucleotides with a Dm resulted in the observation of the single-stranded oligonucleotides; data not shown).

At all three collision energies, the 1:1 $\mathrm{Dm}^{-}$ oligonucleotide duplex dissociates more readily than the 2:1 Dm-oligonucleotide duplex. At higher collision energies, both the 1:1 Dm-oligonucleotide duplex and 2:1 Dm-oligonucleotide duplex also undergo significant fragmentation. For the 1:1 Dm-oligonucleotide duplex, the overall intensity of the most abundant ion decreased by a factor of $\sim 18$. For the $2: 1 \mathrm{Dm}^{-}$ oligonucleotide duplex, the overall intensity of the most abundant ion decreased by a factor of $\sim 5$. The differences observed in Figure 10 indicate that the 2:1 Dm-oligonucleotide duplex is more stable in the gas phase relative to the $1: 1 \mathrm{Dm}$-oligonucleotide duplex. These results appear to reflect solution binding, where the 2:1 Dm-oligonucleotide duplex was previously found to be more stable than the 1:1 Dm-oligonucleotide duplex. The increase in stability may be due to the additional stacking interactions between the two Dm molecules in the minor groove [34]. Both the 1:1 and 2:1 Dm-oligonucleotide duplex noncovalent complexes appear to dissociate less than the bare oligonucleotide duplex (Figure 9a). The stability differences observed between the oligonucleotide duplex (Figure 9a), 1:1 Dm-oligonucleotide duplex (Figure 10a-c), and 2:1 Dm-oligonucleotide duplex (Figure 10d-f) may be related, in part, to the slightly different centerof-mass collision energies that apply for these studies (due to differences in mass-to-charge ratio of the precursors). However, it appears that the present results support a qualitative correlation between the solution and gas-phase stability of these noncovalent complexes.

\section{Conclusions}

The analysis of oligonucleotides for molecular weight and sequence information can be accomplished readily by using ESI-MS. The results presented here demonstrate that ESI-MS and ESI tandem mass spectrometry are effective analytical techniques for the detection of specific minor groove binding molecule-oligonucleo- tide duplex noncovalent complexes. Under appropriate solution and ESI interface conditions, signal intensities for oligonucleotide duplex noncovalent complexes are observed in relatively high abundance. In certain cases, the signals for the noncovalent complex were greater than those observed for the single-stranded oligonucleotide components. Our results demonstrate that only the sequence-specific oligonucleotide duplexes expected in solution are observed in the ESI mass spectra. No nonspecific dimers, trimers, or other aggregation were observed or were of minor contribution under the conditions used. For the two 14-base pair sequence-specific oligonucleotides, the minor groove binding molecule $\mathrm{Dm}$ was observed to be associated specifically with the sequence-specific oligonucleotide duplex, and nonspecific duplexes complexed with $\mathrm{Dm}$ were not observed. These noncovalent complexes have been shown to be dissociated readily in the gas phase into their component parts. The stability of the 2:1 $\mathrm{Dm}$-oligonucleotide duplex in the gas phase was found to be greater than the 1:1 $\mathrm{Dm}$-oligonucleotide duplex at all energies, which is consistent with known solution behavior. The ability to rapidly observe small molecule-oligonucleotide duplex noncovalent complexes by ESI-MS may enable the analysis of minor groove binding molecules with different functional groups, structures, and properties. ESI-MS may also contribute to the study of molecules with enhanced binding to specific DNA sequences. The small concentrations required ( $<5 \mathrm{nmol}$ of noncovalent complex) consumed (generally $<100$ pmol of noncovalent complex), and the relative speed with which experiments can be performed may enable ESI-MS to be used as a specific rapid screening technique prior to detailed experiments utilizing more traditional techniques (two-dimensional NMR, x-ray crystallography).

\section{Acknowledgments}

Pacific Northwest Laboratory is operated by Battelle Memorial Institute for the U.S. Department of Energy through Contract DE-AC06-76RLO-1830. We would also like to thank B. L. Schwartz, D. G. Camp, Il, J. H. Wahl, A. K. Morris, S. L. Delinger, K. J. Light-Wahl, E. C. Sisk, A. F. Fuciarelli, B. D. Thrall, and D. L. Springer for their assistance, and D. R. Goodlett for helpful discussions in the early phases of this work.

\section{References}

1. Whitehouse, C. M.; Dreyer, R. N.; Yamashita, M.; Fenn, J. B. Anal. Chem. 1985, 57, 675-679.

2. Yamashita, M.; Fenn, J. B. J. Phys. Chem. 1984, 88, 2240-2249.

3. Henry, K. D.; Williams, E. R.; Wang, B.-H.; McLafferty, F. W.; Shabanowitz, J.; Hunt, D. F. Proc. Natl. Acad. Sci. U.S.A. 1989, 86, 9075-9078.

4. Henry, K. D.; Quinn, J. P.; McLafferty, F. W. I. Am. Chem. Soc. 1991, 113, 5447-5449.

5. Chowdhury, S. K.; Eshraghi, J;; Wolfe, H.; Forde, D.; Hlavac, A. G.; Johnston, D. Anal. Chem. 1995, 67, 390-398.

6. Reddy, D. M.; Rieger, R. A.; Torres, M. C.; Iden, C. R. Anal. Biochem. 1994, 220, 200-207. 
7. Potier, N.; Dorsselaer, A. V.; Cordier, Y.; Roch, O.; Bischoff, R. Nucleic Acits Res. 1994, 22, 3895-3903.

8. Little, D. P.; Chorush, R. A.; Speir, J. P.; Senko, M. W.; Kelleher, N. L.; McLafferty, F. W. I. Am. Chem. Soc. 1994, $116,4893-4897$.

9. Limbach, P. A.; Crain, P. F.; McCloskey, J. A. I. Am. Soc. Mass Spectrom. 1995, 6, 27-39.

10. Cheng, X.; Gale, D. C.; Udseth, H. R.; Smith, R. D. Anal. Ch'm. 1995, 67, 586-593.

11. Schwartz, B. L.; Light-Wahl, K. J.; Smith, R. D. I. Am. Soc. Mnss Spectrom. 1994, 5, 201-204.

12. Light-Wahl, K. J.; Schwartz, B. L.; Smith, R. D. I. Am. Chem. Soc. 1994, 116, 5271-5278.

13. Li, Y.-T.; Hsieh, Y.-L.; Henion, J. D.; Ocain, T. D.; Schiehser, G. A.; Ganem, B. J. Am. Chem. Soc. 1994, 116, 7487-7493

14. Li, Y.-T.; Hsieh, Y.-L.; Henion, J. D.; Senko, M. W.; McLafferty, F. W.; Ganem, B. I. Am. Chem. Soc. 1993, 115, 8409-8413.

15. Light-Wahl, K. J.; Winger, B. E.; Smith, R. D. J. Am. Chem. Soc. 1993, 115, 5869-5870.

16. Ganem, B.; Li, Y.-T.; Henion, J. D. I. Amt. Ch'm. Soc. 1991, $113,6294-6296$.

17. Ogorzalek Loo, R. R.; Goodlett, D. R.; Smith, R. D.; Loo, J. A. 1. Am. Chem. Soc. 1993, 115, 4391-4392.

18. Hsieh, Y.-L.; Cai, J.; Li, Y.-T.; Henion, J. D.; Ganem, B. J. Am. Soc. Mass Spectron. 1995, 6, 85-90.

19. Light-Wahl, K. J.; Springer, D. L.; Winger, B. E.; Edmonds, C. G.; Camp, D. G., II; Thrall, B. D.; Smith, R. D. J. Am. Chem. Soc. 1993, 115, 803-804.

20. Ganem, B.; Li, Y.-T.; Henion, J. D. Tetrahedron Lett. 1993, 34, $1445-1448$.

21. Bayer, E.; Bauer, T.; Schmeer, K.; Bleicher, K.; Maier, M.; Gaus, H.-J. Anal. Chem. 1994, 66, 3858-3863.

22. Smith, R. D.; Light-Wahl, K. J. Biol. Mass Spectrom. 1993, 22, 493-501.

23. Myers, A. G.; Cohen, S. B.; Kwon, B. M. I. Am. Chem. Soc. 1994, 116, 1255-1271.

24. Roche, C. J.; Thomson, J. A.; Crothers, D. C. Biochemistry 1994, 33, 926-935.

25. Cullinane, C.; van Rosmalen, A.; Phillips, D. R. Biochemistry 1994, 33, 4632-4638.

26. Boger, D. L.; Johnson, D. S.; Yun, W. I. Am. Chem. Soc. 1994, $116,1635-1656$.

27. Van Houten, B.; Illenye, S.; Qu, Y.; Farrell, N. Biochemistry 1993, 32, 11794-11801.

28. Kusakabe, T.; Maekawa, K.; Ichikawa, A.; Uesugi, M.; Sugiura, Y. Biochemistry 1993, 32, 11669-11675.

29. Liu, F.; Meadows, K. A.; McMillin, D. R. J. Am. Chem. Soc. 1993, 115, 6699-6704.

30. Silva, D. J.; Kahne, D. E. I. Am. Chem. Soc. 1993, 115, 7962-7970.

31. Pindur, U.; Haber, M.; Sattler, K. J. Chem. Ed. 1993, 70, 263-272.

32. Geierstanger, B. H.; Jacobsen, J. P.; Mrksich, M.; Dervan, P. B.; Wemmer, D. E. Biochemistry 1994, 33, 3055-3062.

33. Walker, S.; Murnick, J.; Kahne, D. J. Am. Chem. Soc. 1993, 115, 7954-7961.

34. Pelton, J. G.; Wemmer, D. E. I. Am. Chem. Soc. 1990, 112, 1393-1399.

35. Pelton, J. G.; Wemmer, D. E. Proc. Natl. Acad. Sci. U.S. A. 1989, 86, 5723-5727.

36. Fagan, P.; Wemmer, D. E. J. Am. Chem. Soc. 1992, 114, 1080-1081.

37. Kennard, O.; Hunter, W. N. Angew. Chem. Int. Ed. Engl. 1991, 30, 1251-1277.

38. Edwards, K. J.; Jenkins, T. C.; Neidle, S. Biochemistry 1992, 31. 7104-7109.
39. Coll, M.; Aymami, J.; van der Marel, G. A.; van Boom, J. H.; Rich, A.; Wang, A. H.-J. Biochemistry 1989, 28, 310-320.

40. Coll, M.; Frederick, C. A.; Wang, A. H.-J.; Rich, A. Proc. Natl. Acat. Sci. U.S. A. 1987, 84, 8385-8389.

41. Fletcher, M. C.; Fox, K. R. Nucleic Acids Res. 1993, 21 , 1339-1344.

42. Uchida, K.; Pyle, A. M.; Morii, T.; Barton, J. K. Nucleic Acids Res. 1993, 17, 10259-10279.

43. Mrksich, M.; Dervan, P. B. I. Am. Chem. Soc. 1993, 115. 2572-2576.

44. Adnet, F.; Liquier, J.; Taillandier, E.; Singh, M. P.; Rao, K. E.; Lown, J. W. I. Biomol. Struct. Dyntam. 1992, 10, 565-575.

45. Bailly, C.; Colson, P.; Hénichart, J.-P.; Houssier, C. Nucleic Acids Res. 1993, 21, 3705-3709.

46. Parkinson, J. A.; Ebrahimi, S. E.; McKie, J. H.; Douglas, K. T. Biochemistry 1994, 33, 8442-8452.

47. Chu, W.; Kamitori, S.; Shinomiya, M.; Carlson, R. G.; Takusagawa, F. J. Am. Chem. Soc. 1994, 116, 2243-2253.

48. Singh, M. P.; Plouvier, B.; Hill, G. C.; Gueck, J.; Pon, R. T.; Lown, J. W. I. Am. Chem. Soc. 1994, 116, 7006-7020.

49. He, G.-X.; Browne, K. A.; Groppe, J. C.; Blaskó, A.; Mei, H.-Y.; Bruice, T. C. J. Am. Chem. Soc. 1993, 115, 7061-7071.

50. Zimmer, C.; Wähnert, U. Prog. Biophys. Mol. Biol. 1986, 47, 31-112.

51. Kopka, M. L.; Yoon, C.; Goodsell, D.; Pjura, P.; Dickerson, R. E. Proc. Natl. Acad. Sci. U.S. A. 1985, 82, 1376-1380.

52. Dervan, P. B. Science 1986, 232, 464-471

53. Bruice, T. C.; Mei, H.-Y.; He, G.-X.; Lopez, V. Proc. Natl. Acad. Sci. U.S. A. 1992, 89, 1700-1704.

54. Wade, W. S.; Mrksich, M.; Dervan, P. B. Biochemistry 1993. 32, 11385-11389.

55. Watson, J. D.; Hopkins, N. H.; Roberts, J. W.; Steitz, J. A.; Weiner, A. M. Molectular Biology of the Gente, 4th ed.; Menlo Park, CA: Benjamin/Cummings, 1987; pp 149-150, 240-255.

56. Wilson, W. D. In Nucleic Acids in Chemistry and Biology; Blackburn, G. M.; Gait, M. J., Eds.; Oxford, U.K.: IRL Press, 1990; pp 297-303.

57. Mote, J., Jr.; Ghanouni, P.; Reines, D. J. Mol. Biol. 1994, 236 , 725-737.

58. Browne, K. A.; He, G.-X.; Bruice, T. C. J. Am. Chem. Soc. 1993, 115, 7072-7079.

59. Gale, D. C.; Smith, R. D. I. Am. Chem. Soc. 1994, 116, 6027-6028.

60. Hsieh, Y.-L.; Li, Y.-T.; Henion, J. D.; Ganem, B. Biol. Mass Spectrom. 1994, 23, 272-276.

61. Smith, R. D.; Barinaga, C. J.; Udseth, H. R. Anal. Chem. 1988, 60, 1948-1952.

62. Chowdhury, S. K.; Katta, V.; Chait, B. T. Rapid Commun. Mass Spectrom. 1990, 4, 81-87.

63. Gale, D. C.; Smith, R. D. Rapid Communt. Mass Spectrom. 1993, 7, 1017-1021.

64. Wampler, F. M., III; Blades, A. T.; Kebarle, P. J. Am. Soc. Mnss Spectrom. 1993, 4, 289-295.

65. Ding, J.; Anderegg, R. J. I. Am. Soc. Mass Spectrom. 1995, 6, 159-164.

66. Busman, M.; Knapp, D. R.; Schey, K. L. Rapid Commun. Mass Spectrom. 1994, 8, 211-216.

67. Aplin, R. T.; Robinson, C. V.; Schofield, C. J.; Westwood, N. J. J. Chem. Soc., Chem. Commun. 1994, 2415-2417.

68. Hardin, C. C.; Henderson, E.; Watson, T.; Prosser, J. K. Biochemistry 1991, 30, 4460-4472.

69. Hardin, C. C.; Corregan, M.; Brown, B. A., II; Frederick, L. N. Biochemistry 1993, 32, 5870-5880.

70. Goodlett, D. R.; Camp, D. G., II; Hardin, C. C.; Corregan, M.; Smith, R. D. Biol. Mass Spectrom. 1993, 22, 181-183. 
71. Geierstanger, B. H.; Mrksich, M.; Dervan, P. B.; Wemmer, D. E. Scivnte 1994, 266, 646-650.

72. Chen, Y.-H.; Lown, J. W. I. Am. Chem. Soc. 1994, 116, 6995-7005.

73. Mrksich, M.; Dervan, P. B. I. Am. Chom. Soc. 1994, 116. 3663-3664.

74. Mrksich, M.; Dervan, P. B. I. Am. Chem. Soc. 1993, 115, 2572-2576
75. Mrksich, M.; Dervan, P. B. I. Am. Chem. Soc. 1993, 115, 9892-9899.

76. Dwyer, T. J.; Geierstanger, B. H.; Bathini, Y.; Lown, J. W.; Wemmer, D. E. I. Alr. Cli'm. Soc. 1992, 114, 5911-5919.

77. Wade, W. S.; Mrksich, M.; Dervan, P. B. I. Am. Chem. Soc. 1992, 114, 8783-8794. 